\title{
Three-Dimensional Impedance-Based Sensors for Detection of Chemicals in Aqueous Solutions
}

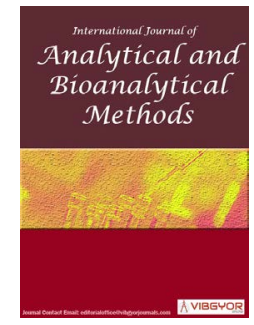

\section{Nadia E Tolouei', Roya Mazrouei ${ }^{1,2}$ and Mohammad Shavezipur ${ }^{{ }^{*}}$}

\author{
${ }^{1}$ Department of Mechanical and Mechatronics Engineering, Southern Illinois University, USA \\ ${ }^{2}$ Stanford Genome Technology Center, Stanford University, USA
}

\begin{abstract}
In this work, the development of three-dimensional impedance-based biochemical sensors for detection of chemicals and biological agents in aqueous solutions is presented. The sensors are made of a stack of suspended electrodes that allow the solution to occupy the space between them and create a larger interface area between the aqueous solution and the electrodes. Increasing the solution-electrode interface area drastically changes the impedance of the sensor-solution resulting in a better sensitivity. Low concentrations of di-ethylhexyl phthalate (DEHP) in deionized water are used as the target chemical to demonstrate the advantage of new design over conventional planar interdigitated sensors. Experimental measurements are carried out to characterize the response of the planar and 3D sensors and their Nyquist plots are compared, displaying significant sensitivity improvement in 3D sensors. An electrical model for the sensors is developed that considers different physical phenomena such as doublelayer capacitance, solution resistance, Warburg effect and parasitic parameters. Nonlinear interpolations of the experimental data show that the equivalent electrical circuit is in good agreement with the Nyquist plots obtained from test data. The curve fitting of the tested data to the equivalent electrical circuit displays good agreement between the model and the tested data.
\end{abstract}

\section{Keywords}

MEMS biochemical sensors, Chemical detection, Electrochemistry Impedance Spectroscopy (EIS), Interdigitated sensors, Three-Dimensional biochemical sensors, di-ethylhexyl phthalate (DEHP)

\section{Introduction}

Detection of biological agents (cell, enzymes, proteins, etc.) and chemicals in many critical areas has been revolutionized by progression in microelectronics and nanotechnology and also by exploiting new materials such as carbon nanotubes (CNT) and graphene. The combination of miniatur- ized sensors and microfluidic systems has led to the development of lab-on-a-chip systems for a wide range of applications from point-of-care and cancer detection to food safety and environmental studies [1-3]. Different biochemical sensing methods have been developed using advancement in micro electromechanical systems (MEMS)and microelec-

*Corresponding author: Mohammad Shavezipur, Department of Mechanical and Mechatronics Engineering, Southern Illinois University, Edwardsville, IL, USA

Accepted: August 19, 2020; Published: August 21, 2020

Copyright: ( 2020 Tolouei NE, et al. This is an open-access article distributed under the terms of the Creative Commons Attribution License, which permits unrestricted use, distribution, and reproduction in any medium, provided the original author and source are credited.

Tolouei et al. Int J Analyt Bioanalyt Methods 2020, 2:012 
tronics technology, such as inertial sensors [4], field-effect-transistors [5], and impedance-based interdigitated electrodes [6]. FET-based sensors use the change in characteristic response of the device in presence of a chemical or biological agent for detection $[7,8]$. Micro-beams with electrostatic actuation are used as inertial sensors to detect cells, enzymes and proteins with suitable surface modifications that allow the target agent to adhere to the beam surface and change its frequency response $[9,10]$. Impedance-based sensors are also widely used for biochemical detection because of their simple structure and manufacturing process, ease of use and high accuracy for detection at extremely low concentrations, where the target biomarker or chemical is presented to the sensor in aqueous solution or suspension [11,12]. All these sensing transduction methods have demonstrated excellent detection capability with high sensitivity and can be used at sub-ppm concentrations, with high sensitivity.

One advantage of using electrochemistry impedance spectroscopy (EIS) is the ability to provide information about electrochemical properties of the solution such as ion transportation, electron transfer, diffusion phenomena and the double-layer effect [13-15]. The combination of an impedance-based sensor and the aqueous solution is often modeled by an equivalent circuit that can define the behavior of the sensor-solution system [16]. The electrical circuit simulating the sensor-solution includes electrical elements that represent different physical phenomena. For example, the double-layer effect is presented by double-layer capacitance [17], and the diffusion phenomenon near the electrode surface is quantified by Warburg impedance [18].

In this work, we report the development of novel impedance-based biochemical sensors with three-dimensional structures where one of the electrodes (or both) are suspended in the solution. Suspended electrodes have been used in the past for gas detection in field-effect-transistor (FET) and capacitive sensors [19-22]. The sensors used in this work have a different geometry and work in aqueous media to detect chemicals and biological agents such as cells and bacteria. This geometric modification increases electrode-solution interface area and hence, improves the sensitivity of the sensor to the change of the solution concentration. Larg- er electrode-solution interface area directly affects parameters such as double-layer capacitance and Warburg impedance, and therefore creates more significant change in the Nyquist response of the sensor-solution system when the solution concentration changes. To demonstrate the advantage of proposed design over existing planar interdigitated sensors, we use low concentrations of di-ethylhexyl phthalate (DEHP) in water. DEHP imposes health hazards and has been used in the past for demonstration of electrochemical impedance spectroscopy for interdigitated sensors, and provides a platform to compare the performance of the proposed $3 D$ sensor with existing 2D sensors. In the next sections, the design concept, fabrication process, measurement setup and results are presented.

\section{Sensors Design and Fabrication Process \\ Design concept}

Conventional impedance-based biochemical sensors consist of two conductive interdigitated electrodes (IDE) patterned on a substrate, as shown in Figure 1a. Depending on the fabrication process, the substrate may be non-conductive (like glass), or conductive (like single-crystal silicon) which requires an insulator layer to electrically separate the sensors and substrate. Different conductive materials such as metals and doped polycrystalline silicon have been used in the past to fabricate planar interdigitated sensors $[13,15,18,23]$. The working principle of planar (2D) IDE biochemical sensors is based on the interaction between the electrodes and the aqueous solution (or suspension) at their interface and throughout the solution medium. An IDE sensor, exposed to an aqueous medium, acts like an electrical circuit comprised of a combination of parallel and series electrical elements with certain impedances, where each element represents a physical phenomenon. For example, the positive and negative electrical charges, accumulated on the surface of the electrodes and nearby solution, create the double-layer capacitance $[17,24]$, or the diffusion phenomenon happens in the solution medium near the surface of electrodes at low frequencies is represented by Warburg impedance $[25,26]$. If the composition of the solution changes, then the values of these parameters change and allow for the detection of targeted chemical or biomarker in the solution. Depending on the fabrication process, each sensor may also add constant capacitive and resistive elements to the electrical circuit of 


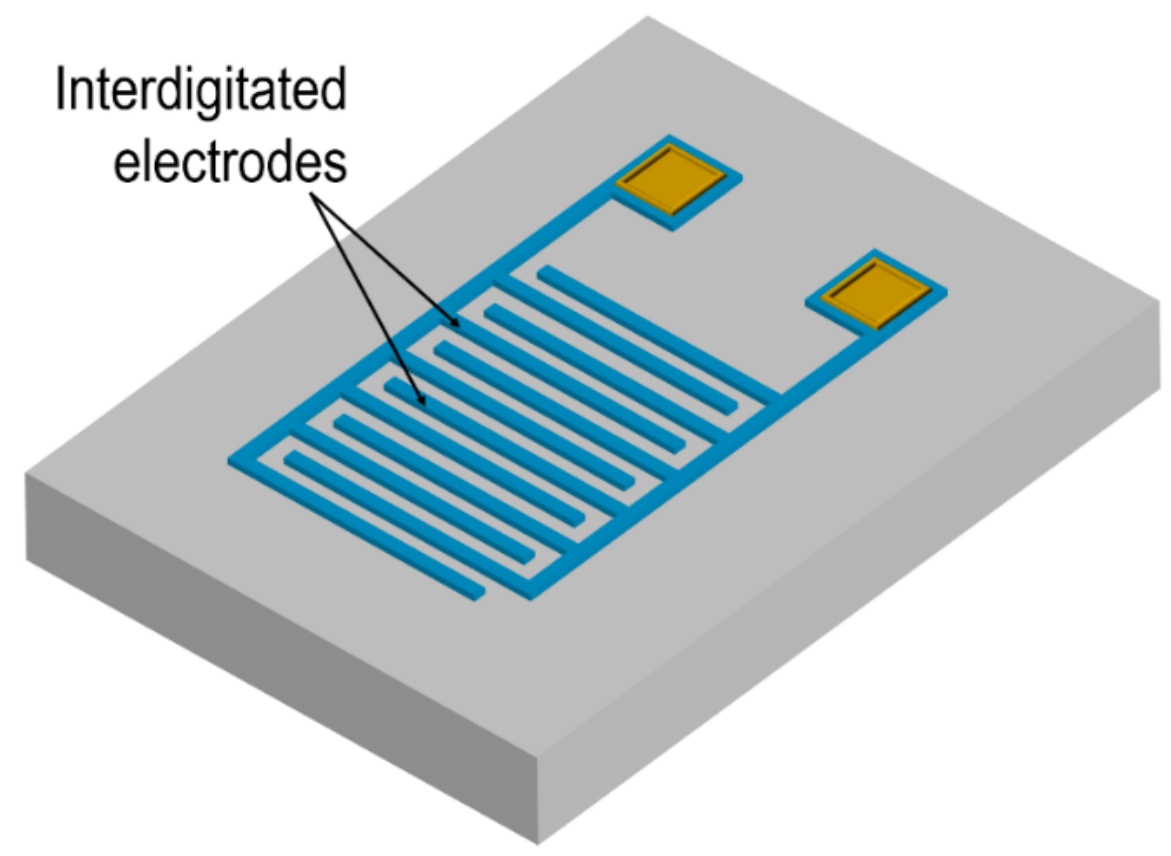

a)

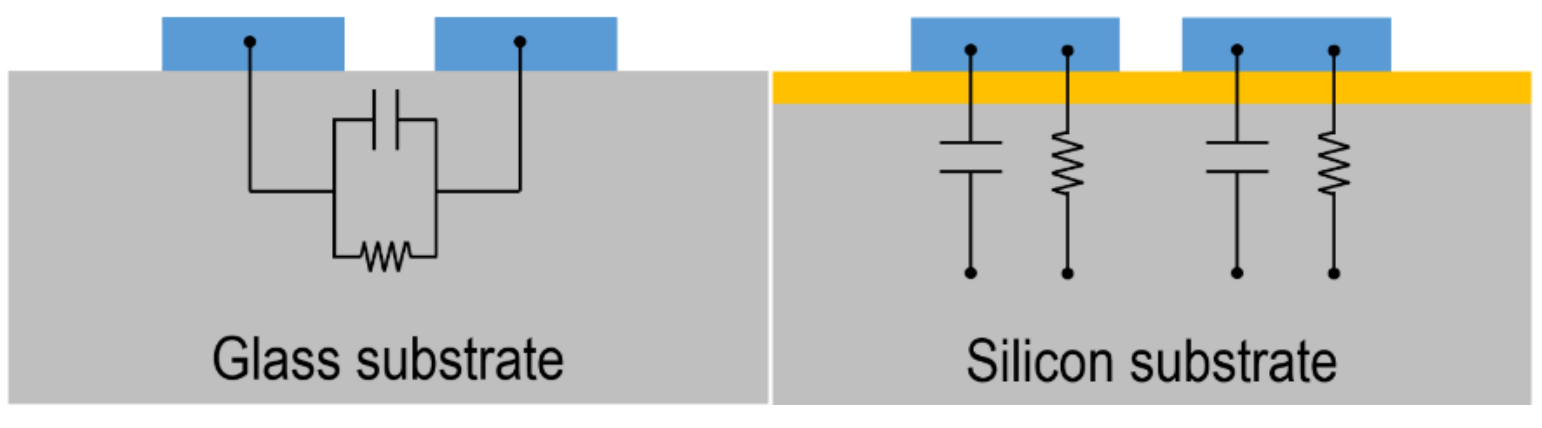

b)

c)

Figure 1: (a) A conventional two-dimensional IDE impedance-based biochemical sensor; (b) Parasitic capacitance and resistance elements between IDE and glass substrate; and (c) Parasitic capacitance and resistance elements between IDE and silicon substrate $[27,29]$.

the system [27]. Since these constant parameters do not depend on the solution composition and mainly depend on the sensor structure (Figure $1 \mathrm{~b}$ and Figure 1c), they act as parasitic elements and their presence may negatively affect the sensor's response and reduce its sensitivity. The main parasitic parameters are the capacitive and resistive values between the electrodes and the substrate, the resistance value of the transmission lines that connect the devices to the measurement equipment, and the capacitive and resistive values between the transmission line/contact pads and the substrate $[28,29]$.

Considering the major parameters that affect the detection process are the solution dependent (dynamic) electrical elements and all these ele- ments rely on the electrode-solution interface, it is expected that if the effective interface area is increased, then a higher sensitivity is achieved. The sensors presented in this work are designed to have a higher effective electrode-solution interface area compared to planar IDEs. The proposed sensor designs have 3D structures where one or both of the sensing electrodes are suspended and the aqueous solution can reach both sides of the electrodes creating a larger electrode-solution interface. It should be noted that it is possible to increase the interface area for conventional IDEs by using larger electrodes, but this also increases the parasitic capacitance/resistance values shown in Figure $1 \mathrm{~b}$ and Figure 1c. Therefore, larger IDE sensors may provide a larger impedance readout, but they do not 


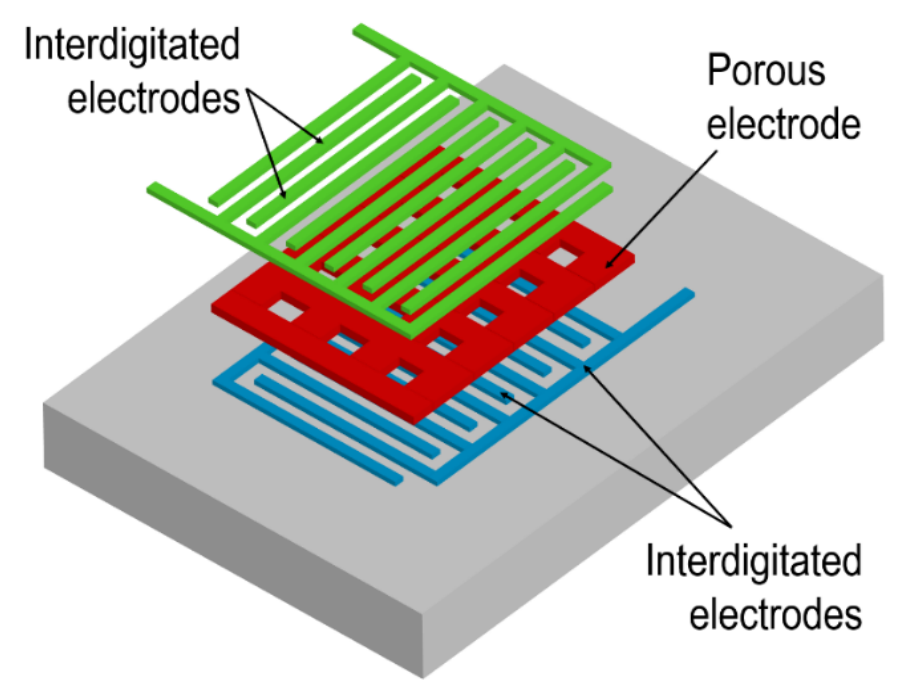

a)

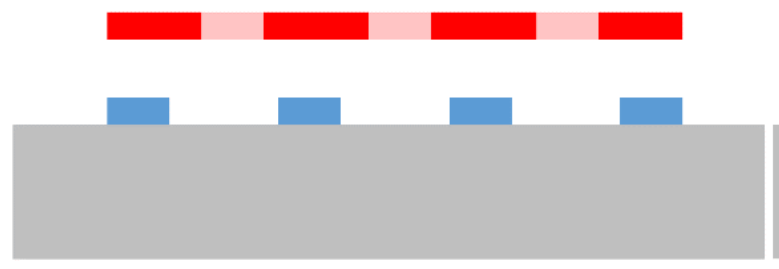

b)

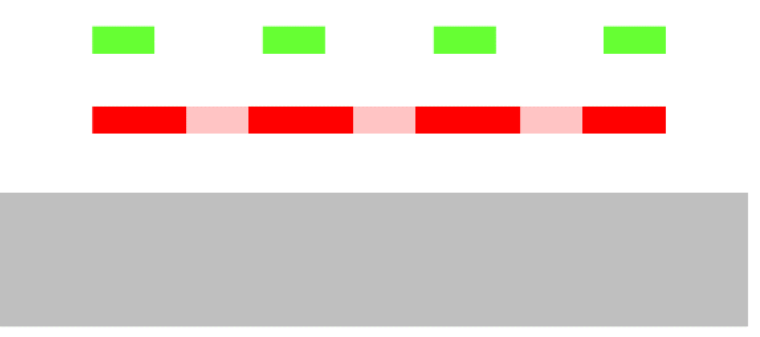

c)

Figure 2: (a) Three-dimensional impedance-based biochemical sensor with three layers of sensing electrodes; (b) One patterned, and one suspended electrode configuration; and (c) Two suspended electrodes configuration.

necessarily improve the sensitivity of the sensor response. The $3 \mathrm{D}$ design modification results in a larger interface area compared to the planar design using the same electrode size and do not add the parasitic capacitive/resistive elements. Considering the sensors are suspended in the solution, the new design even reduces the parasitic capacitance/resistance values compared to the conventional IDE, because the suspended sensors have much smaller physical contact with the substrate, mainly at anchors that hold the suspended structures. Other parasitic elements related to the transmission lines and contact pads remain unchanged in both $2 \mathrm{D}$ and $3 \mathrm{D}$ designs. Figure $2 \mathrm{a}$ illustrates the design idea, where the stack of three electrode layers for a 3D structure is schematically shown. The pair of each two layers would form a set of impedance-based sensor, as shown in Figures $2 b$ and Figures $2 c$. The design presented in this work uses two sets of IDE for top and bottom electrodes and a perforated plate for the middle electrode. Two electrodes in each set of IDE are short-circuited and act as one electrode, so the 3D sensor practically has three electrodes in chemical sensing (separate IDEs at each level are designed for biological applications to detect the cell attachment to the surface which is not in the scope of this work).

\section{Fabrication process}

A standard micromachining process is used to fabricate the planar IDE and 3D biochemical sensors, called PolyMUMPs. The process uses polycrystalline silicon as the conductive and structural layer and silicon dioxide as the sacrificial layers. The devices are manufactured on a single-crystal silicon wafer. The wafer and conductive layers are electrically isolated using a $0.6 \mu \mathrm{m}$ thick silicon nitride thin film. Details of the fabrication process, electrical and mechanical properties of the polysilicon and sacrificial layers, and the mask design rules can be found in [30]. Figure 3 displays the fabrication process of the planar IDE sensors and 3D biochemical sensors. The electrode patterned on the substrate (that also forms 2D IDE) is made by Polyo layer, the middle electrode is made by Poly1 layer, and the top electrode is made using Poly 2 layer. The thick- 


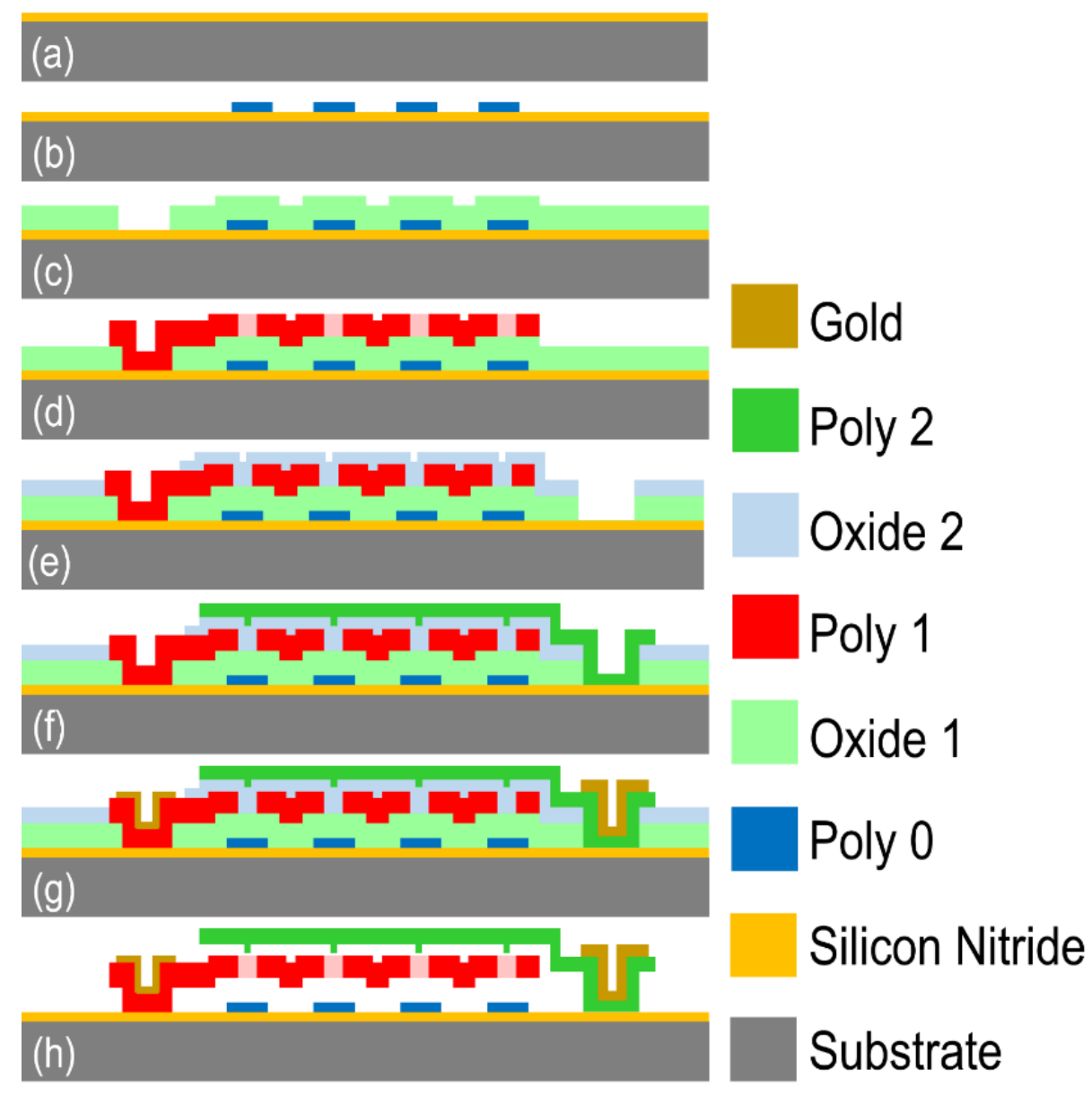

Figure 3: Fabrication process of planar IDE and three-dimensional biochemical sensors. Details of the process including the thickness of structural and sacrificial layers and mask design rules can be found in process handbook [30].

ness of Poly0, Poly1 and Poly2 layers is $0.5 \mu \mathrm{m}, 2.0$ $\mu \mathrm{m}$ and $1.5 \mu \mathrm{m}$, respectively, and the thickness of sacrificial layers Oxide1 and Oxide2 is $2.0 \mu \mathrm{m}$ and $0.75 \mu \mathrm{m}$, respectively, and the top gold layer is 0.5 $\mu \mathrm{m}$ thick. Planar IDE are fabricated using only steps (a) and (b) of the process, and their contact pads are coated with gold.

Figure 4 presents the SEM images of fabricated sensors. A planar IDE sensor is shown in Figure 4a, and a 3D sensor is presented in Figure $4 \mathrm{~b}$ and Figure $4 c$, and both sensors have an overall size of 400 $\times 400 \mu^{2}$. The anchors for each set of suspended electrodes (Poly1 and Poly2 layers) are at two sides of the sensor. As shown in close-up image of the 3D sensor in Figure 4c, the conformality of the deposited thin films in fabrication process (Figure 3 ) resulted in wavy-shape middle and top electrodes, and the electrodes are not completely flat in the areas that the bottom layer has holes. These geometric irregularities do not affect the electrochemical interaction between the electrodes and the solu- tion. However, if needed, they can be eliminated by adding a chemical-mechanical polishing (CMP) step after deposition of each sacrificial layer.

\section{Test Setup}

One planar IDE sensor and two 3D sensors are tested and the measurement setup is shown in Figure 5 . The chip that contains the sensors is tested on a probe station equipped with a microscope. The impedance is recorded for each signal frequency using a Keysight E4980A Precision LCR meter, and the sensors are connected to the LCR meter via the probe tips and co-ax cables to minimize the noise. A small rectangular container, made by a 3D printer, is glued to the surface of the chip to separate the solution from probe pads (as illustrated in Figure 5b). All probe pads are fabricated at the edge of the chip and are connected to the sensors via long transmission line.

For chemical detection, di-ethylhexyl phthalate (DEHP) is used as a target chemical and is dissolved 


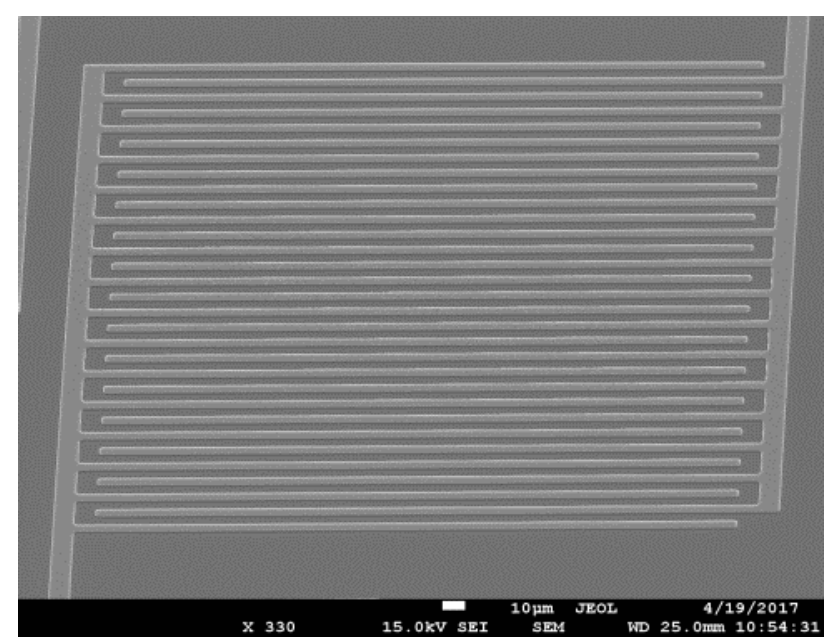

a)

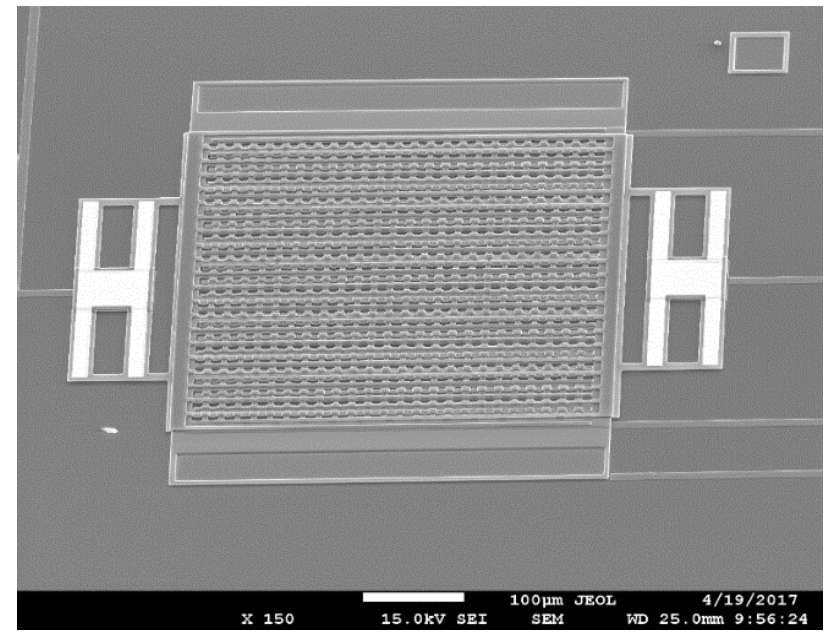

b)

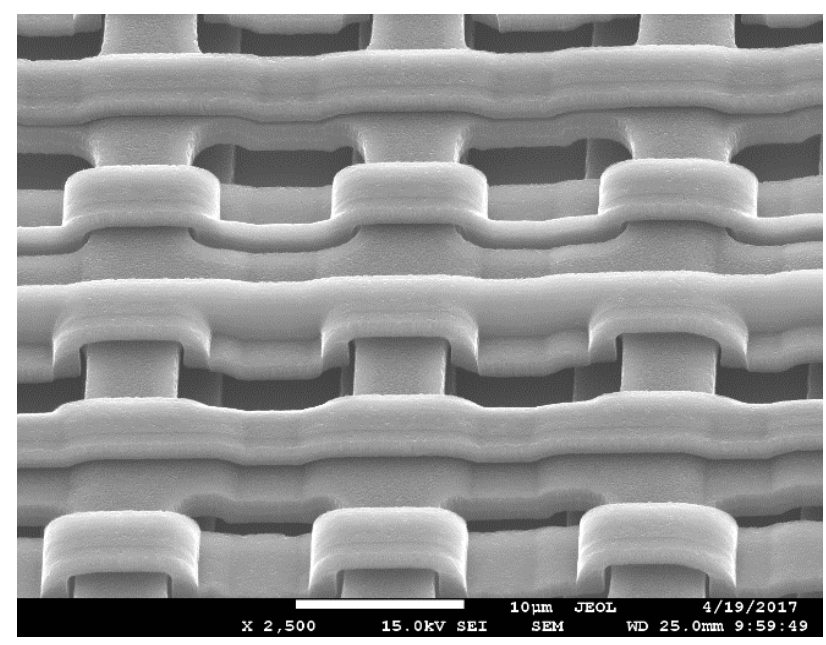

c)

Figure 4: SEM images of fabricated biochemical sensors: (a) Planar IDE sensor, the width and the gap between the fingers is $5 \mu \mathrm{m}$ and the overall size of the sensor is $400 \times 400 \mu^{2}$; (b) A three-dimensional sensor with electrode configuration similar to the one schematically presented in Figure 2. in deionized water at low concentrations. Phthalates are commonly used in food packaging and have a high potential for non-occupational leaching and ingestion into packaged beverages such as bottled water, juice and soda drinks and they are directly absorbed into human body fluids [15]. DEHP solution has been used in the past for characterization of biochemical sensors $[15,29]$. Low concentrations of the DEHP solution are prepared by first, adding ethanol (2 ppm) to the deionized water as a polar solvent to dissolve DEHP, and then DEHP is added to the distilled water and different concentrations of DEHP solutions, from $0.02 \mathrm{ppm}$ to $10 \mathrm{ppm}$ are prepared for impedance measurements. The devices are tested by sweeping the signal frequency from $10 \mathrm{kHz}$ to $2 \mathrm{MHz}$, and the system impedance are recorded and stored using a MATLAB ${ }^{\circledR}$ interface program. The recorded impedance values are used to plot the Nyquist responses of the sensors for different DEHP concentrations, as will be discussed in the next section.

\section{Measurement Results}

To investigate the performance of the sensors, the Nyquist plots of each sensor are plotted for different concentrations of DEHP and compared in Figure 6. In general, electrochemical impedance measurement for IDE sensors exposed to aqueous solutions results in two distinct regions in Nyquist plot: A straight line at low frequency and a half circle at high frequencies [31-33]. The sensors' responses presented here display both regions and differentiate between concentrations of DEHP, demonstrating the ability to capture even very low concentrations of the targeted chemical for chemical detection. However, there are major differences between the response of 2D planar sensor and the $3 \mathrm{D}$ sensors as described in the following.

A closer look at the Nyquist plots presented in Figure 6 shows that the responses of 3D sensors for different concentrations are more separated and distinct than those of the 2D sensor. This is specially more visible in Figure $6 \mathrm{c}$ and for the 3D sensor that has two suspended electrodes. This distinction in the Nyquist response is associated with the larger electrode-solution interface area for the 3D sensors compared to the 2D planar IDEs that increases the dynamic (solution-dependent) impedance values. Increasing the interface area between the electrodes and the solution increases dynamic parameters such as double layer capaci- 


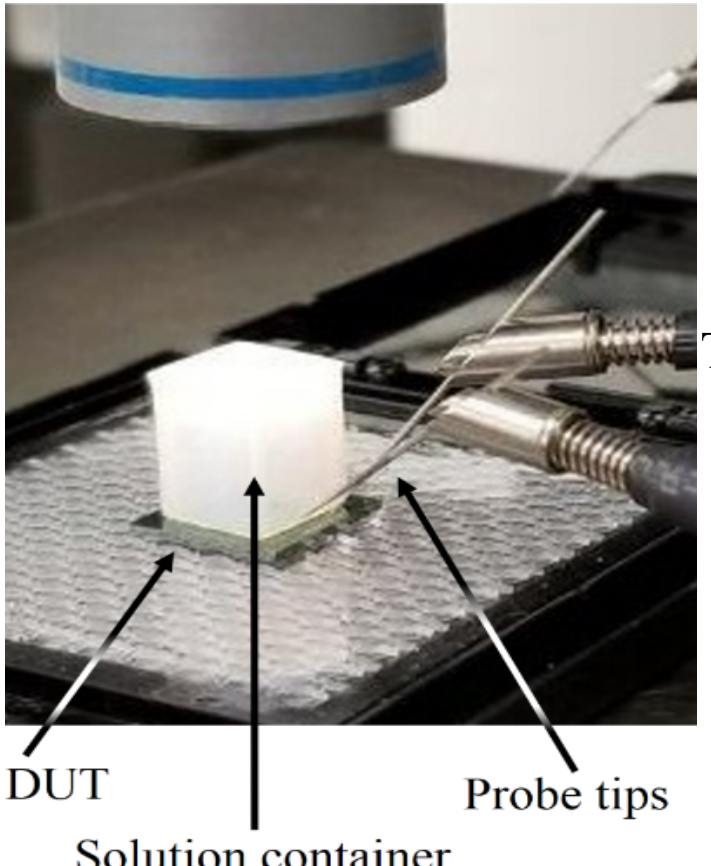

Solution container

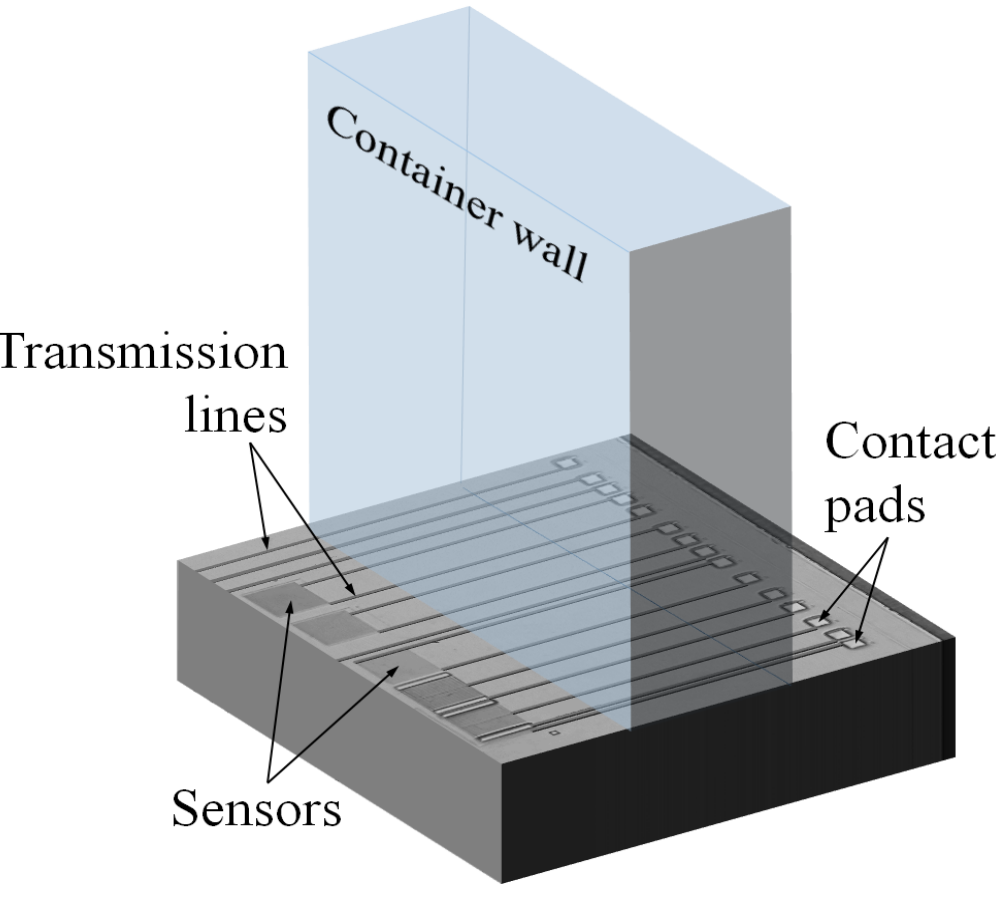

b)

Figure 5: (a) Experimental set up including the chip with the fabricated device, the solution container and probe tips and micro-positioners; (b) Schematic of the sensor, container wall and contact pads configuration. The 3D printer solution container separates the sensors from the contact pads.

tance, $C_{d \prime}$ and Warburg impedance, $Z_{w}$. Comparing the magnitude of the real and imaginary impedances in conventional IDE sensor and 3D sensors show that the impedance for planar electrodes is much smaller than those of the 3D sensors, and the 3D sensor with two suspended electrode has the highest impedance values, where for low concentration test $(0.02 \mathrm{ppm})$, the system impedance at low frequency exceeds $1 \mathrm{M} \Omega$. Another parameter that contributes to the increases in system impedance is the use of a plate as the middle electrode. The middle (plate) electrode has much larger surface area compared to the comb-shape electrodes and therefore provides much larger interface with the solution, resulting in much larger $C_{d l}$ and $Z_{w}$ values. Moreover, the parasitic capacitive and resistive values decrease compared to the 2D planar sensor, creating a larger relative change in the impedance of the system.

For quantitative analysis of the sensors' responses, the equivalent circuit of the sensors is studied. Different electrical circuits have been suggested in the literature for IDE sensors used for biochemical detection $[16,22,26,34,35]$. To consider different physical parameters contributing to the performance of the sensors, such as $C_{d l}, Z_{w}$ and parasit- ic capacitive and resistive elements, an equivalent electrical circuit is suggested to simulate the sensor-solution behavior. Figure $7 \mathrm{a}$ and Figure $7 \mathrm{~b}$ show the schematic of different elements in the sensing system and the equivalent circuit associated with them, respectively. In the equivalent electrical circuit, $R_{T 1,2}$ represent the resistance of the transmission lines connetcing the electrodes to the contact pads. $C_{\text {sub } 1,2}$ and $R_{\text {sub } 1,2}$ are the capacitance and the resistance between the electrodes (including the anchors, transmission line and contact pads) and the substrate $[29,36] . R_{\text {sol }}$ is the solution resistance and $C_{d 11,2}$ and $Z_{w 1,2}$ represent the double-layer capacitance and Warburg impedance at the interface of the two electrodes and the solution, respectively. $R_{d i 1,2}$ and $C_{d i 1,2}$ represent the resistance and capacitance created on the surface of the polysilicon electrodes due to the formation of a thin silicon dioxide film on theelectrodes surface. We have shown that the formation of native oxide affects the diffusion (Warburg) effect since there is a thin dielectric layer on the surface of the electrodes [35]. The thin (2-3 nm) dielectric layer formed on the electrode surface separates the electrode (the conductvie part) from the solution and this separation is modeled by $C_{d i}$ and $R_{d i}$. 


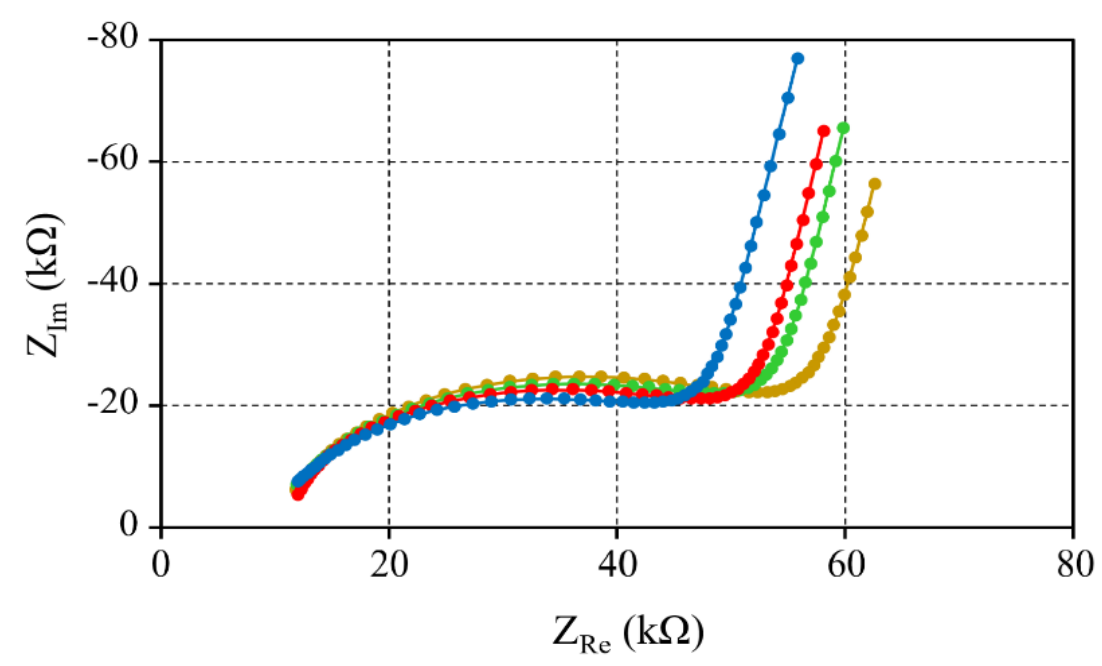

a)

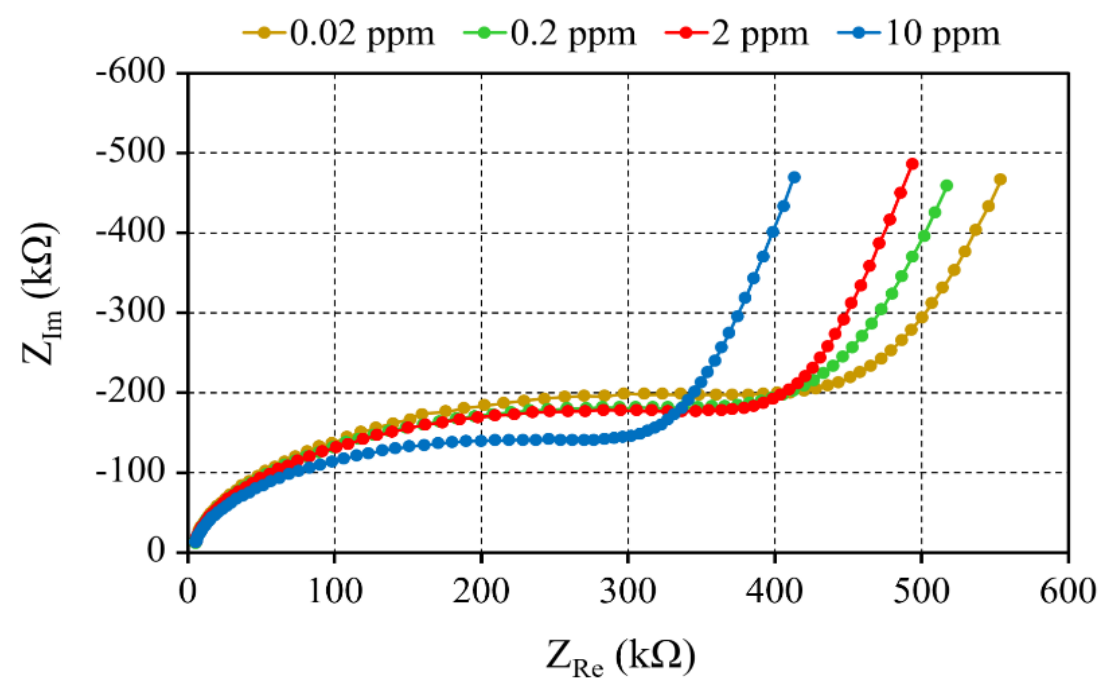

b)

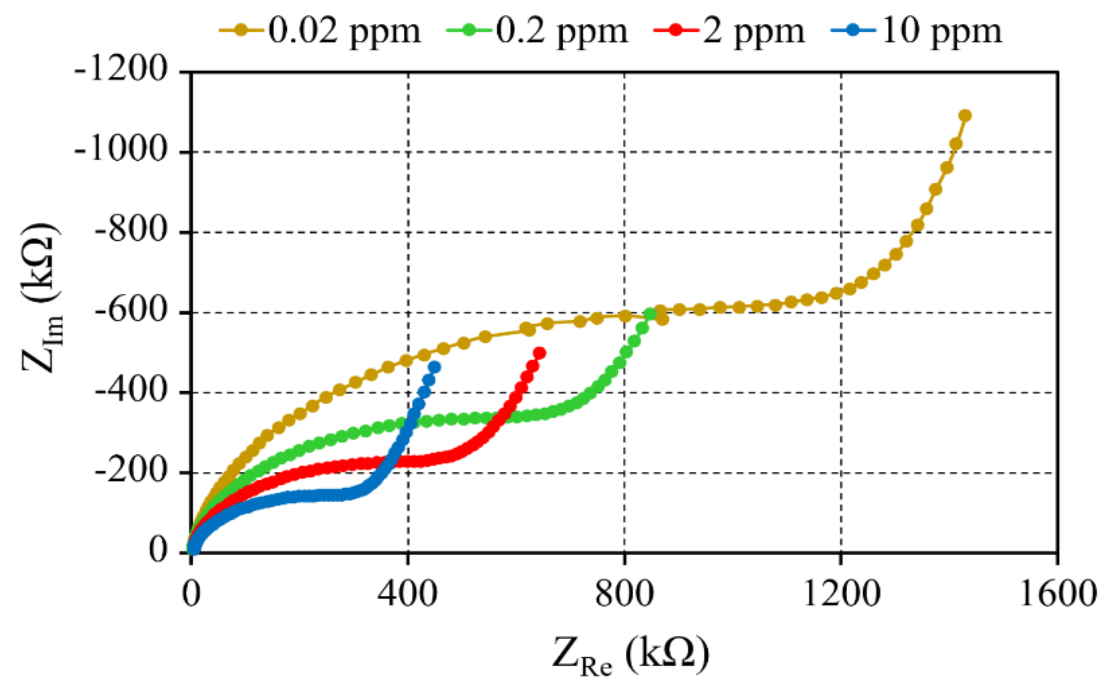

c)

Figure 6: Nyquist diagrams for: (a) Conventional (planar) IDE sensor; (b) Three-dimentional sensor with one electrode suspended electrode and one electrode patterned on the substrate; and (c) Three-dimentional sensor with two suspended electrodes. 


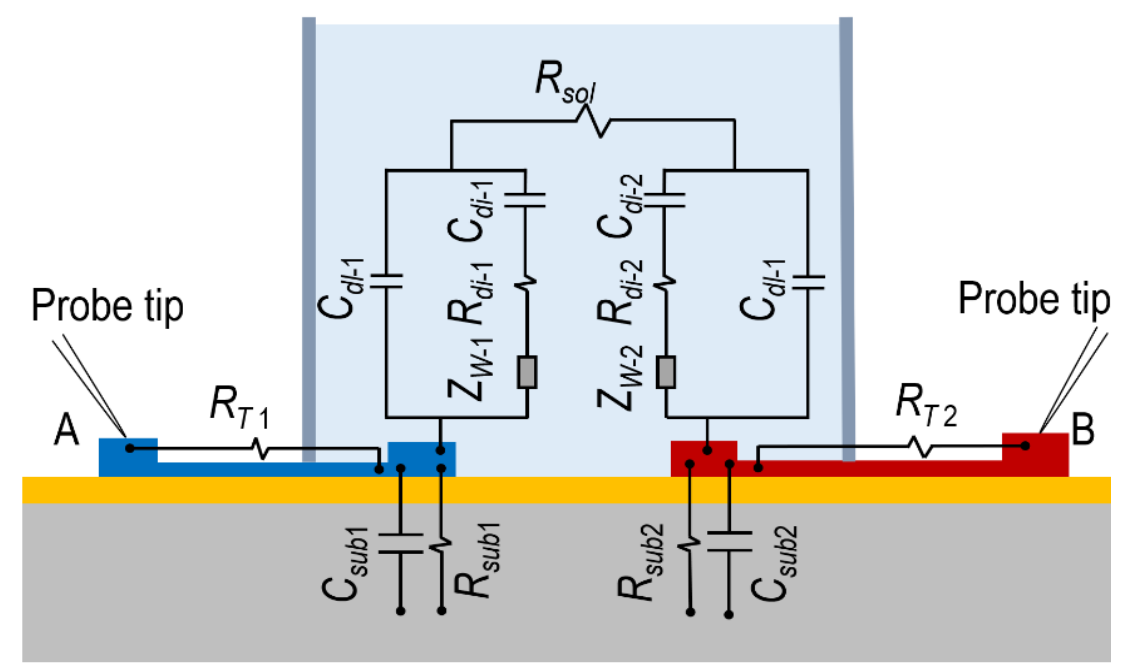

a)

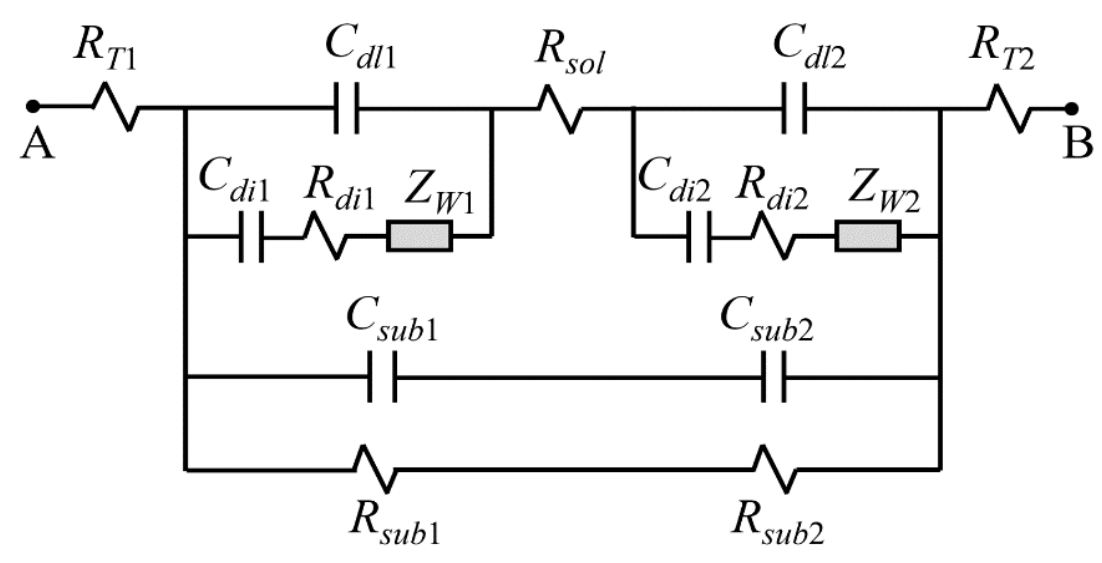

b)

Figure 7: (a) Physical elements that contribute to the electrochemical response of the biochemical sensors; and (b) The equivalent electrical circuit represnting the sensor and the solution.

The electrical model and the parameters shown in Figure 7a are used for 2D and 3D sensors, as the fundamental functions all sensors are the same. The parasitic elements in the equivalent circuit are $R_{\text {T1,2, }} C_{\text {sub1,2, }}, R_{\text {sub1, } 2^{\prime}} R_{\text {di1,2 }}$ and $C_{d i 1,2}$ and the dynamic elements are $C_{d l^{\prime}} A_{w}$ and $R_{\text {sol }}$, where $A_{w}$ is the Warburg coefficient is obtained from:

$$
Z_{W}=\frac{A_{W}}{\sqrt{\omega}}+\frac{A_{W}}{j \sqrt{\omega}}
$$

And $\omega$ represents the signal frequency. As explained before, the values of parasitic elements only depend on the sensor geometry and electrode sizes; however, the dynamic parameters such as $C_{d l}$ and $A_{w}$ are expected to be larger in 3D sensors, because they depend on the electrode-solution interface area. To examine the equivalent electrical circuit and the values of dynamic parameters, we use one concentration for all three sensors to fit a curve to the experimental data using a specific program developed for curve fitting for electrochemical impedance spectroscopy [36]. Figure 8 presents the results of curve fitting using the equivalent circuit of Figure $7 \mathrm{~b}$ and the data for concentration of $2 \mathrm{ppm}$. The curve fitting for all three responses display acceptable match, where the $2 \mathrm{D}$ sensor has the best match of the three tested sensors.

The three-dimensional biochemical sensors presented in this work provide high sensitivity and can be used to detect low concentrations of chemicals and biomarkers. The scope of the work presented here was limited to chemical sensing; however, the sensors can be also used for biological sensing to detect cells, proteins, enzyme and other biomarkers in aqueous solutions. It should be noted that electrochemical impedance spectroscopy better serves the biological sensing because for cell, bacteria or protein detection, the selectivity of the sensor can 


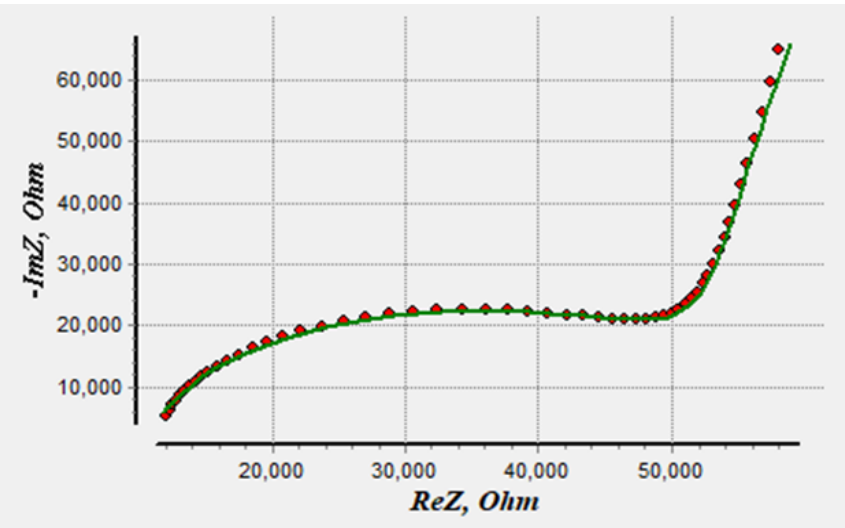

a)

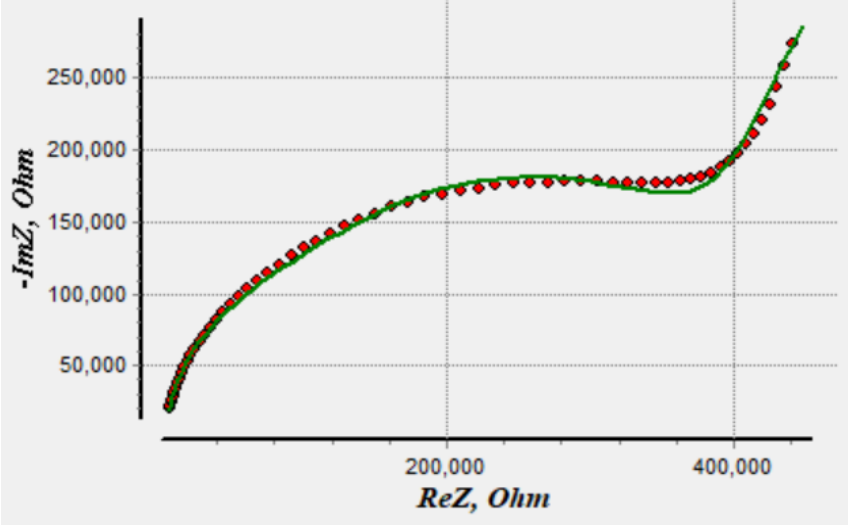

b)

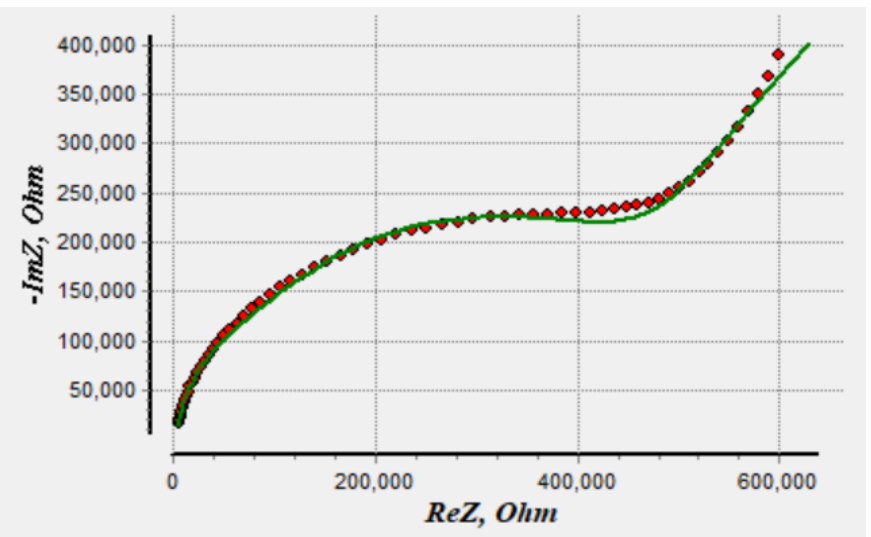

c)

Figure 8: Nonlinear interpolation fitting the equivalent circuit to the experimental Nyquist data obtained for 2 ppm DEHP concentration of 2D and 3D sensors: (a) 2D planar IDE sensor; (b) 3D sensor with one fixed and one suspended electrode (Poly0-Poly1); and (c) 3D sensor with two suspended electrodes (Poly1-Poly2).

be improved using electrodes surfaces modified by antibody or DNA/RNA strand associated with the target agent. This allows the electrodes to attract the target biological agent to their surface and create bonds with them. However, for detection in an aqueous medium, the presence of contamination for biological and chemical targets affects the accuracy of the sensing method. The sensors presented in this work may not improve the selectivity, but under similar circumstances provide better sensitivity compared to the 2D planar IDEs. Moreover, using other conductive materials such as metals with no dielectric coating on the electrode surface eliminates some of the parasitic elements exist in polysilicon-based electrodes, and could further improve the sensor sensitivity.

\section{Concluding Remarks}

This work presented three-dimensional impedance-based biochemical sensors. The 3D sensors use suspended electrodes that have interface with solution through their top and bottom surfaces, doubling the interface area. Larger solution-electrode interface area allows the electrolyte solution to interact with a larger electrode surface, and results in larger values in solution-sensitive parameters such as double-layer capacitance and Warburg coefficient, and therefore improves the sensitivity of the sensor. Moreover, the 3D sensors have smaller parasitic (constant) capacitance and resistance values which further improve the sensitivity of the sensor to the change in solution concentration. Comparing the performance of one conventional 2D planar interdigitated sensor and two 3D sensors using different concentrations of di-ethylhexyl phthalate (DEHP) in de-ionized water shows that the 3D sensors provide distinctly higher sensitivity. Higher sensitivity allows the sensors to detect a slight change in solution concentration, and can be used for biological measurements that require detection of a small change in the sample concentration. 


\section{Acknowledgments}

The authors acknowledge the financial support by Southern Illinois University, Edwardsville, under "Seed Grants for Transitional and Exploratory Projects (STEP)".

\section{References}

1. Mehrotra P (2016) Biosensors and their applications-A review. J Oral Biology and Craniofacial Research 6: 153-159.

2. Pol R, Céspedes F, Gabriel D, Baeza M (2017) Microfluidic lab-on-a-chip platforms for environmental monitoring. TrAC Trends in Analytical Chemistry 95: 62-68.

3. Huang X, Tang C, Xu Q, Zhao Y, Li D (2017) Microfluidic "Lab-on-a-Chip" sensing in food safety and quality analysis. Sensing Techniques for Food Safety and Quality Control, 61-94.

4. Perlmutter $M$, Breit $S$ (2016) The future of the MEMS inertial sensor performance, design and manufacturing. DGON Intertial Sensors and Systems (ISS), 1-12.

5. Schöning MJ, Poghossian A (2002) Recent advances in biologically sensitive field-effect transistors (BioFETs). Analyst 127: 1137-1151.

6. Sacco A (2017) Electrochemical impedance spectroscopy: Fundamentals and application in dye-sensitized solar cells. Renewable and Sustainable Energy Reviews 79: 814-829.

7. Schütz $S$, Schöning MJ, Schroth $P$, Malkoc Ü, Weissbecker B, et al. (2000) An insect-based BioFET as a bioelectronic nose. Sensors and Actuators B: Chemical 65: 291-295.

8. Barrios CA (2009) Optical slot-waveguide based biochemical sensors. Sensors 9: 4751-4765.

9. Raiteri R, Grattarola M, Butt HJ, Skládal P (2001) Micromechanical cantilever-based biosensors. Sensors and Actuators B: Chemical 79: 115-126.

10.Ziegler C (2004) Cantilever-based biosensors. Anal Bioanal Chem 379: 946-959.

11.Varshney M, Li Y (2007) Interdigitated array microelectrode-based impedance biosensor coupled with magnetic nanoparticle-antibody conjugates for detection of Escherichia coli 0157: H7 in food samples. Biosensors and Bioelectronics 22: 2408-2414.

12.Yang L, Li Y, Erf GF (2004) Interdigitated array microelectrode-based electrochemical impedance immunosensor for detection of Escherichia Coli 0157: H7. Anal Chem 76: 1107-1113.
13. Randviir EP, Banks CE (2013) Electrochemical impedance spectroscopy: An overview of bioanalytical applications. Analytical Methods 5: 1098-1115.

14.Lasia A (2002) Electrochemical impedance spectroscopy and its applications. Modern Aspects of Electrochemistry, Springer, Boston, USA, 143-248.

15.Zia Al, Syaifudin AM, Mukhopadhyay SC, Al-Bahadly IH, Yu PL, et al. (2011) Development of Electrochemical Impedance Spectroscopy based sensing system for DEHP detection. Fifth IEEE International Conference on Sensing Technology, 666-674.

16.Chen CC, Huang BC, Lin MS, Lu YJ, Cho TY, et al. (2010) Impedance spectroscopy and equivalent circuits of conductively doped organic hole-transport materials. Organic Electronics 11: 1901-1908.

17.Uddin MS, Das HT, Maiyalagan T, Elumalai P (2018) Influence of designed electrode surfaces on double layer capacitance in aqueous electrolyte: Insights from standard models. Applied Surface Science 449: 445-453.

18.Yuan X, Xu N (2001) Determination of hydrogen diffusion coefficient in metal hydride electrode by modified Warburg impedance. Journal of Alloys and Compounds 329: 115-120.

19.Blackburn G, Janata J (1982) The suspended mesh ion selective field effect transistor. Journal of The Electrochemical Society 129: 2580-2584.

20.Cassidy J, Pons S, Janata J (1986) Hydrogen response of palladium coated suspended gate field effect transistor. Anal Chem 58: 1757-1761.

21. Mlsna TE, Cemalovic S, Warburton M, Hobson ST, MIsna DA, et al. (2006) Chemicapacitive microsensors for chemical warfare agentand toxic industrial chemical detection. Sensors and Actuators B 116: 192-201.

22.Lazarus N, Fedder GK (2011) Integrated vertical parallel-plate capacitive humidity sensor. J Micromech Microeng 21: 065028.

23. De La Rica R, Fernández-Sánchez C, Baldi A (2006) Polysilicon interdigitated electrodes as impedimetric sensors. Electrochemistry Communications 8: 1239-1244.

24.Kleppisius A, Kick A, Mertig M (2017) Double-layer capacitance of $\mathrm{pH}$-sensitive self-assembled thiol layers on gold determined by electrical impedance spectroscopy in a microfluidic channel. Physica Status Solidi(a) 214: 1600921.

25. Huang J (2018) Diffusion impedance of electroactive materials, electrolytic solutions and porous electrodes: Warburg impedance and beyond. Electrochimica Acta 281: 170-188. 
26. Barbero G, Lelidis I (2017) Analysis of Warburg's impedance and its equivalent electric circuits. Physical Chemistry Chemical Physics 19: 24934-24944.

27.Paredes J, Becerro S, Arizti F, Aguinaga A, Del Pozo JL, et al. (2013) Interdigitated microelectrode biosensor for bacterial biofilm growth monitoring by impedance spectroscopy technique in 96-well microtiter plates. Sensors and Actuators B: Chemical 178: 663670.

28. Mazrouei R, Minako S, Mohammad S (2018) Detection of pathogens and formation of biofilms using a three-dimensional biomimetic biosensing platform. ASME Conference: American Society of Mechanical Engineers Digital Collection.

29. Mazrouei R, Huffman B, Sumita M, Shavezipur M (2019) Development of an impedance-based interdigitated biochemical sensor using a multiuser silicon process. Journal of Micromechanics and Microengineering 29: 075011.

30.Cowen A, Hardy B, Mahadevan R, Wilcenski S (2011) PolyMUMPs design handbook. Memscap Inc, 13.

31. Macdonald JR, Barsoukov E (2005) Impedance spectroscopy: Theory, experiment, and applications. History 1: 1-13.
32.Sathya S, Muruganand S, Manikandan N, Karuppasamy K (2019) Design of capacitance based on interdigitated electrode for BioMEMS sensor application. Materials Science in Semiconductor Processing 101: 206-213.

33.Rivadeneyra A, Salmerón JF, Agudo-Acemel $M$, Capitan-Vallvey LF, López-Villanueva JA, et al. (2018) Asymmetric enhanced surface interdigitated electrode capacitor with two out-of-plane electrodes. Sensors and Actuators B: Chemical 254: 588596.

34. MacDonald MA, Andreas HA (2014) Method for equivalent circuit determination for electrochemical impedance spectroscopy data of protein adsorption on solid surfaces. Electrochimica Acta 129: 290-299.

35.Ebrahimpour TN, Ghamari S, Shavezipur M (2020) Investigation of the effect of native oxide layer on performance of interdigitated impedance-based silicon biochemical sensors. ASME Int Design Eng Tech Conf, USA, 5.

36. Murbach MD, Hu VW, Schwartz DT (2017) The impedance analyzer: An open-source, Web-based tool for sophisticated interrogation of experimental EIS spectra. ECS Meeting Abstracts 46: 2035-2035. 